\title{
Rabaska
}

Revue d'ethnologie de l'Amérique française

CORGERON, JEAN-MICHEL, LAURENT JARRY, LISE BEAUCHEMIN, FRANK SEARS et SÉBASTIEN DESHAIES. Musique traditionnelle québécoise pour accordéon diatonique. 20 thèmes avec variations et ornements. Répertoire sélectionné et interprété par FRANK SEARS. Partitions et tablatures par JEAN- MICHEL CORGERON. Aulnay-sous-Bois, Éditions Franches Connexions, 2018, 2 vol. (vol. 1, 64 p. ; vol. 2, 66 p.). 2 DVD audio et vidéo. ISBN 2-9517073-5-5 et 2-9517073-4-7

\section{Steve Normandin}

Volume 17, 2019

URI : https://id.erudit.org/iderudit/1066026ar

DOI : https://doi.org/10.7202/1066026ar

Aller au sommaire du numéro

Éditeur(s)

Société québécoise d'ethnologie

ISSN

1703-7433 (imprimé)

1916-7350 (numérique)

Découvrir la revue

Citer ce compte rendu

Normandin, S. (2019). Compte rendu de [CORGERON, JEAN-MICHEL, LAURENT Jarry, Lise Beauchemin, Frank SeArs et SÉbastien Deshaies. Musique traditionnelle québécoise pour accordéon diatonique. 20 thèmes avec variations et ornements. Répertoire sélectionné et interprété par FRANK SEARS. Partitions et tablatures par JEAN- MICHEL CORGERON. Aulnay-sous-Bois, Éditions

Franches Connexions, 2018, 2 vol. (vol. 1, 64 p. ; vol. 2, 66 p.). 2 DVD audio et vidéo. ISBN 2-9517073-5-5 et 2-9517073-4-7]. Rabaska, 17, 293-295.

https://doi.org/10.7202/1066026ar d'utilisation que vous pouvez consulter en ligne. 
Corgeron, Jean-Michel, Laurent Jarry, Lise Beauchemin, Frank Sears et SÉBASTIEN Deshaies. Musique traditionnelle québécoise pour accordéon diatonique. 20 thèmes avec variations et ornements. Répertoire sélectionné et interprété par Frank Sears. Partitions et tablatures par JeanMichel Corgeron. Aulnay-sous-Bois, Éditions Franches Connexions, 2018, 2 vol. (vol. 1, 64 p. ; vol. 2, 66 p.). 2 Dvd audio et vidéo. ISBN 2-9517073-5-5 et 2-9517073-4-7.

Préserver et perpétuer la tradition orale des musiques par lesquelles les Québécois ont su se bâtir un folklore tout en permettant aux musiciens de tous les horizons (de l'autodidacte au croque-notes) de s'initier à ce vaste répertoire, c'est une forme de mission à laquelle plusieurs musiciens se prêtent sous des formes différentes : sites internet (les initiatives du violoniste Pascal Gemme ou le site Identit'Airs géré par Paul Dubé, entre autres), quelques rares livres de partitions (parmi les pertinences : Carmelle Bégin et Pierre Chartrand pour le recueil rare sur les compositions de l'accordéoniste Philippe Bruneau ou le splendide Danse ce soir réalisé en version anglais/français par deux Américains passionnés, Laurie Hart et Greg Sandell, aux Éditions Mel Bay). Toutes ces initiatives sont louables et nécessaires... mais elles se font rares, en comparaison avec toute la musique enregistrée!

L'oralité est de mise dans la transmission et la diffusion du répertoire ancien et contemporain. Il ne faut pas se le cacher : pour les musiciens qui ont le coup de cœur pour notre « swing » et la beauté des mélodies, le niveau d'exécution de notre répertoire peut rebuter. Si vous avez une bonne oreille et de la patience, tout va ; mais lorsque vous avez appris à lire la musique, ou que vous devez ralentir le mouvement pour vous imprégner d'une mélodie à danser, si vous n'avez pas de professeur disponible pour vous enseigner, que faites-vous? Vous cherchez de possibles méthodes.

Le Centre Mnémo, dirigé par Pierre Chartrand et son équipe dynamique, a remis, en 2018, son vingtième Prix Mnémo pour honorer un ouvrage colossal et admirable. Deux bouquins qui apportent un éclairage technique sur l'art de jouer ces mélodies en se rapprochant du style (pour les passionnés d'accordéon à une rangée, en premier lieu). Rien ne manque : ornementations diverses, autour d'une même mélodie, tant à l'écrit qu'à l'image. Partition dans le ton d'origine de l'accordéon utilisé par Sears (souvent sur un accordéon en ré) et sa transposition en do pour les musiciens du diatonique européen (plus spécifiquement dans le milieu du bal folk en France). Tour de force et travail d'orfèvre. Ces partitions et leurs tablatures sont rédigées par le Français Jean-Michel Corgeron, génialement secondé par les musiciens québécois que sont l'accordéoniste Frank Sears et la pianiste Lise Beauchemin.

Jean-Michel Corgeron fut un des artisans de la toute première heure à Trad Magazine, défunt magazine français, dans lequel il proposait des 
transcriptions de différents répertoires retracés dans les nombreux folklores des régions de France. Lui-même harmoniciste et accordéoniste, il développe au fil de ses années de publication le « système Corgeron », tablature unique qui facilite la compréhension du poussé-tiré caractéristique de l'accordéon diatonique lors de la lecture d'une partition. Fort de cette expérience, il signe depuis 1987 plus d'une vingtaine d'ouvrages, non seulement pour le compte de Trad Magazine ou différents éditeurs, mais aussi pour sa maison d'éditions « Franches Connexions ». Tous sont additionnés d'un disque audio ou d'un Dvd.

La volonté de transmettre et de rendre accessibles au plus grand nombre les pièces françaises du répertoire traditionnel ne peut qu'imposer le respect. Corgeron s'est épris de ce que les Français appellent le " mélodéon », l'accordéon à une rangée, sorte d'harmonica à soufflet dont les Québécois et les Cadiens font bon usage. Curieux des folklores annexes se rapportant à cet instrument, Corgeron s'était également « payé la traite » en publiant l'année précédente le même genre d'ouvrage en deux volumes, pour un répertoire tout aussi riche et exigeant : la musique irlandaise. En Irlande, l'accordéon à deux rangées si/do fait loi; parfois, des anciens reprennent le petit accordéon. Jean-Michel Corgeron a collaboré avec un spécialiste en la matière, l'accordéoniste français Gilles Poutoux, qui enflamme ce répertoire en le jouant sur l'accordéon à une seule rangée ; il se rapproche ainsi, dans sa démarche, du travail imposant de Frank Sears dans sa version Québec.

Pour les accordéonistes de tout crin qui voudront se pencher sur l'ouvrage, il faudra se familiariser avec une littérature dense et ô combien efficace : si la méthode est simple à mettre en pratique, le musicien doit se l'approprier. Tout est expliqué (p. 6-10), avec dessins à l'appui ; dans les deux volumes : même texte, même approche avec explication de la méthode de travail.

Les pièces jouées par le duo Sears-Beauchemin impressionnent par leur grâce et pourraient faire l'objet d'un disque supplémentaire tant leurs versions nous animent. Ce qui fait le trésor de ce document, c'est la précision avec laquelle Frank Sears joue le tout au ralenti. L'angle de prise de vue permet d'admirer la finesse de chaque pièce : il n'est pas besoin d'être accordéoniste ou musicien pour apprécier ces versions lentes pour leurs évidentes qualités musicales. Jean-Michel Corgeron s'est allié un des plus grands accordéonistes québécois de l'heure et leurs pédagogies rassemblées donnent une furieuse envie d'apprendre.

Bien que la matière première de ces livres provienne du répertoire québécois, elle est accessible à tous ceux qui veulent se pencher sur le sujet. Il n'en demeure pas moins que les principaux utilisateurs de ce livre feront partie du fidèle lectorat de Jean-Michel Corgeron (français, pour la majorité d'entre eux) 
et de ceux qui fréquentent les bals folk européens. La transmission du savoir se fait aussi par quelques pistes qui auraient pu être évoquées en de courtes biographies : « répertoire d'Alfred Montmarquette, Jos Bouchard, Henri Lacroix, Joseph Guilmette, Marcel Messervier », peut-on lire sous presque chaque titre. Morts ou vivants ? Pièce de source irlandaise ou écossaise ou française, de musique militaire américaine ou européenne?

C'est un parti pris qui, personnellement, me laisse sur ma faim. Toutefois, Corgeron tend la main vers notre curiosité naturelle en indiquant l'adresse du site internet de la Bibliothèque nationale du Canada, Le Gramophone virtuel, à la fin de son avant-propos, afin d'y découvrir les versions originales enregistrées au temps du 78 tours. Triste et petit constat devant un si bel ouvrage : si ces noms résonnent aux oreilles des passionnés de musique québécoise des deux côtés de l'Atlantique, leur mémoire se perd irrémédiablement. Indiquer que ces pièces proviennent de leur répertoire, c'est essentiel ; sans repère biographique, ce ne sont que des ronds dans l'eau. Imaginez pour le commun des mortels... Les recueils de musique irlandaise souffrent de cette même lacune. Le répertoire se veut riche, leur provenance est évoquée, mais toujours en superficie. Le livre Danse ce soir, évoqué précédemment, possédait ce petit plus qui rattachait à une personnalité, à une époque. Ce n'est pas de faire de cet outil précieux une encyclopédie surchargée (là n'est pas son but évident), mais, devant tant de détails musicaux bien présentés, trois ou quatre lignes en bas de page auraient suffi pour évoquer ceux dont le nom est mentionné et qui, au début du $\mathrm{Xx}^{\mathrm{e}}$ siècle, ont marqué par leurs enregistrements ou leur légende les bases uniques de ce qui constitue désormais le patrimoine musical québécois.

Ce n'est pas un bien méchant bémol : un tel niveau musical du point de vue de la notation n'avait jamais été réalisé ou pensé comme tel par des musiciens québécois. L'idée et l'entière réalisation, nous la devons à un cousin français. À sa passion et à son goût de communiquer la beauté des musiques traditionnelles. Tout d'un bloc et le cœur sur la main.

Steve Normandin

Saint-Quay Perros, France

Dubé-Lindsay, Laurence. Journal d'une femme de gardien de phare. Ille Verte, 1934. Publié sous la direction de JoCELYN LindSAY, [Île Verte], chez l'auteur, 2018, 131 p. ISBN 978-2-98176-030-2.

Cette parution nous fait vivre la quotidienneté de l'année 1934 au phare de l'Île Verte par la belle plume de Laurence Dubé-Lindsay, épouse du gardien Freddy Lindsay. L'édition a été orchestrée par son fils Jocelyn, fier 